\title{
OBACIS Phase III: Accreditation and Grading Sheets (AGSs) — The Excel-App
}

\author{
Mohamed A. Ismail, \\ Industrial Systems Engineering, University of Regina, \\ mohamed.ismail@uregina.ca
}

\begin{abstract}
In this paper, an Excel Add-in for automating grade recording and graduate attributes assessment at the course level is presented. Course learning outcomes, accreditation units (AU) input parameters and some other course-specific related data are documented as well. A set of student performance reports are generated and are utilized for closing the loop of the continuous improvement activities mandated by the new CEAB accreditation process. The add-in or the Xl-App is one of the three major constituents of the OBACIS framework. The other two are the Windows application or the Win-App; for accreditation administration operations and the web tool or the Web-App; for data compilation reporting process. The Win-App parse the data collected by the add-in (presumably collected via the Web-App as xlsx files or XML files) and integrate them with other program and faculty-level performance assessment and continuous improvement activities. In addition to the role for which it was created, the xl-App can emit the data collected to suit the learning management systems grade books and web marking systems.
\end{abstract}

Keywords: Graduate Attributes, Outcome-Based Assessment, Accreditation Software, Engineering Education

\section{INTRODUCTION}

Graduate Attributes (GA) Assessment and Continuous Improvement (CI) activities are the two most important features that distinguish the current $\mathrm{CEAB}$ accreditation process from its previous one. Canadian institutions are working diligently to meet the new requirements. Due to the overwhelming effort needed, universities are dedicating much time for related activities, investing in new specialized positions and trying to figure out the most appropriate software tools. The process is still locked in the data collection phase. According to EGAD's survey conducted in 2016 [1], most Canadian programs have not yet reached the point of analyzing the data collected, improving programs curricula and closing the continuous improvement loop. The process expenses might become excessive. The last review of the University of Alberta's ( $U$ of A) nine programs required the collection of more than a ton of documents and consumed approximately 16,000 hours in preparation time involving personnel cost of well over $\$ 1 \mathrm{M}[2]$. Software tools are direly needed to facilitate the process, cut down its costs and streamline its workflow.

Kaupp and Frank [3-5] classified the related education software tools into four major categories: learning management system (LMS), learning content management system (L/CMS), assessment platform (AP), analytics system (AS) and curriculum mapping tool (CMT). Despite the abundance of those general systems, OBA-oriented software is rare to find; most institutions are developing their own systems to have the process under control and meet the CEAB requirements. Brennan et al. [6] have used Excel forms for linking course learning outcomes to course assessments activities. Student achievement reports have been generated and utilized for continual improvement activities. The EGAD project [7] team at Queens University have integrated Rstudio, community software and some other open source elements to create a system for collecting, analyzing, reporting and visualizing graduate attributes [8]. The framework's rich graphics and comparative results is a good step in the right direction of analyzing the data collected and closing the continuous improvement loop. During OBACIS' first phase of development, we introduced a tri-mapping and reporting system using an Excel Add-in; the Xl-App, for graduate attributes, learning outcomes and categorical analytics [9]. The add-in was driven from the Win-App environment and its functionality was limited to the tri-reporting system.

In this paper, we will present the Xl-App as a full-fledged add-in that could be distributed to faculty members to be used for grade reporting automation and collecting all accreditation relevant data. The new add-in is enabled with specialized tab dedicated to grade recording and graduate assessment tools, see Figure 1. The add-in creates a well-protected structure that can be parsed later by the Win-App for program and faculty levels analytics and continuous improvement initiatives.

The Xl-App is designed to meet the following design goals:

1. Automate the Grade Recording Process.

2. Map Graduate Attributes to Course Assessment Tools.

3. Collect extra information needed for Course Information Sheets.

4. Generate Students/Course Performance Reports.

5. Import/export data from/to education information systems such as LMS and AS.

CEEA17; Paper 172

University of Toronto; June 4-7, 2017 
In this paper, we will describe briefly the structure of the OBACIS Accreditation and Grading Sheets (AGS) and how to use them to save much time needed for grading and accreditation reporting. Section 2 describes the major structural elements: assets, AGS gallery, and reporting system. Section 3 introduces the user-friendly assessment tree builder and graduate attributes/learning outcomes (GA/LO) mapping cascading forms. Section 4 summarizes and concludes the paper.

\section{THE OBACIS AGS STRUCTURE}

OBAICS is designed to save time, effort and engage faculty in the new OBA process. Professors and top administration's time is valuable and should be geared towards more value-added activities. As a result, cutting some time from grade compilation and grade recording was an opportunity to improve the process workflow. We consider grade recording as a precursor of most OBA assessment activities. In [9] we introduced the OBACIS block and grade assessment trees, which we will describe here in brief. In [10] we introduced the OBACIS catalogs and the Auto-Generated Course Information sheets. Learning outcomes and accreditation units data are collected to augment the system we have developed in [10].

All course assessment activities are defined by the assessment tree forms. These forms define the course assessment categories and their individual grade items. Individual graduate attributes with their assessment indicators and learning outcomes could be mapped simultaneously. The tree building and GA/LO mapping are done in a cascading fashion. The process starts from the Xl-app using a dedicated ribbon tab called "OBACIS AGSs", see Figures 1 and 2. AGSs stands for Accreditation and Grading Sheets. In OBACIS first draft [9], we called them "Auto Grading



Figure 1: OBACIS AGSs Tab

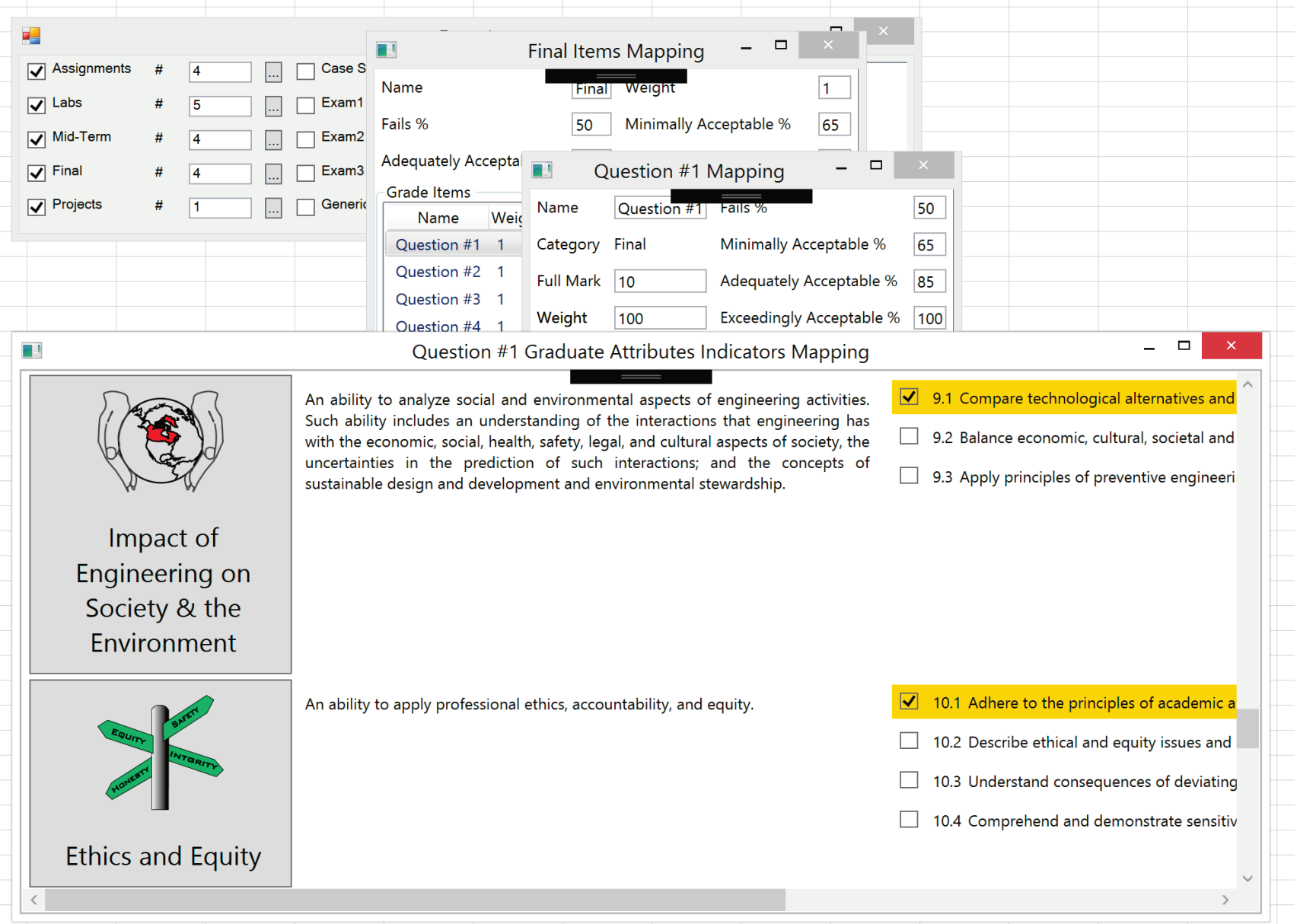

CEEA17; Paper 172

Figure 2: Tree Builder and GA/LO Mapping Cascading Forms 
Sheets". The AGSs tab is composed of three main groups: 1) Assets 2) the Graduate Attributes Gallery 3) The Reports.

\subsection{The Assets}

\section{Students}

The assets group is the place to start. A "Students" button is the place where students can be read. Students can be read



Fiqure 3: Students List

from an excel sheet within the workbook itself after copying it from another workbook or from an XML file. See Figure 3 for a sample students' list.

\section{Learning Outcomes (LO)}

CEAB questionnaire of 2016 made learning outcomes section a mandatory section of the new course information sheets. In [10], we proposed the 'Catalogs' framework to keep updating CIS on a regular basis as a part of a grand program continuous improvement process. In addition, using the course tree, we can map course grade items; those items are called assessment tools by $\mathrm{CEAB}$, to learning outcomes.

\section{Assessment Tree Builder and GA/LO Mapper}

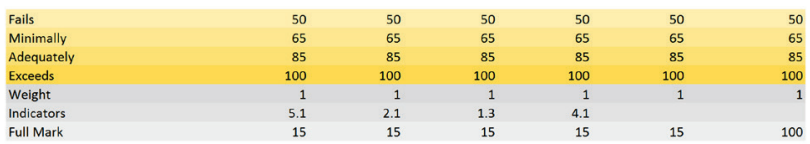

Figure 4: OBACIS Block

Assessment tree is the place that define the entire structure of the workbook and defines the OBACIS block described in [9], see Figure 4.

OBCIS Block defines grade descriptors recommended by CEAB for graduate attributes assessment $[11,12]$. Descriptors are defined for every grade item or parent category. Their values default to $50 \%, 65 \%, 85 \%$ and $100 \%$ to set the boundaries for fails to meet, minimally meets, adequately meets and exceeds expectations descriptors respectively. Weights could have any integer or fractional values. Numbers do not need to add up to 1 . There is a full mark for every item, every category and the entire course. The formula for doing the grade calculations, summations, totals and grand totals are all done automatically. There is an Excel sheet for every category. A separate totals worksheet is defined to sum up all the results. The totals worksheet is done automatically. Using AGSs, all that is needed is just to enter individual grades or copy them for another as a data range that might have been recorded by a teaching assistant.
The XL-App provides a user-friendly assessment tree builder and GA/LO mapper that can be accessed using the "Assessment Tree" button, see Figure 1. The builder/mapper defines course grading hierarchy and maps graduate attributes/ learning outcomes to course assessment tools.

While building the tree, any grade item could be easily mapped to one or more learning outcomes. The same can be done for graduate attributes and their indicators. See section 3 for a detailed demonstration.

\section{Textbooks and AU data}

The new CIS specs [11] require course textbooks list in a certain format. Since we have developed a dedicated framework for the auto-creation of the course information sheets in [10] and have recommended updating them on a regular basis, course textbooks are required to be checked for any updates. AU data such ES, ED, CS are reported for the same purpose as well.

\subsection{Graduate Attributes Gallery}

The graduate attributes and indicators group lists the 12 graduate attributes mandated by CEAB, see Figure 1. Every attribute is defined as a split toggle button. If a graduate attribute indicator has been mapped to a certain grade item, both the indicator and its parent graduate attributes will be toggled on. Toggling indicators and their associated graduate attributes on/off is done through the interactive GA/LO mapping process.

\subsection{OBACIS Reports: The Tri-Reporting System}

The last group of the AGSs tab is the reports group. Three reports are generated: Categorical Analysis (CA) Report, Learning Outcomes Analysis (LOA) Report and OutcomeBased Analysis (OBA) Report. Detailed bar charts and comparative boxplots are generated. All the details of the OBACIS reporting system are presented in [9] for the interested reader. Both bar charts and comparative box plots are generated for every category or mapped GA/LO item. We believe that comparative box plots and bar charts give better signs for continuous improvement opportunities at the course level, See Figures 5 and 6.

\section{TREE BUILDER AND GA/LO MAPPING WALKTHROUGH}

Defining the assessment tree is the only major task required to be done by the teaching faculty. Once done, an empty template could be handed out to the teaching assistants so they can record the grades. In that template, every category has its corresponding OBAICS block. Underneath the block, a table with students' names, IDs and different grade items are formatted and prepared for data entry, see Figure 7. The totals column is equipped with the right formula and is protected against inadvertent access. Any time grades are updated, everything is updated including the 'Totals' worksheet. If there are no grades entered, the totals are zeros. Reports need

CEEA17; Paper 172 
to be triggered first by the user. Once triggered, their updates are done instantaneously as well. Grades can be exported as CSS, XML, or XLSX formats. In the future, the Web-App, not developed yet, will be used for downloading data-rich templates of AGSs. These templates will have student lists, learning outcomes, textbook and other data to be just reviewed by the teaching faculty for any updates. AGSs are supposed to be uploaded to the Win-App to be persisted in the Central Database for program and faculty-wide analytics. The generated XLSX is supposed to be uploaded using the Webapp or using a generalized email to be processed later by the Win-App for program level and faculty level analytics. The estimated savings of using the Xl-App for grade and accreditation reporting could be between $40-50 \%$.

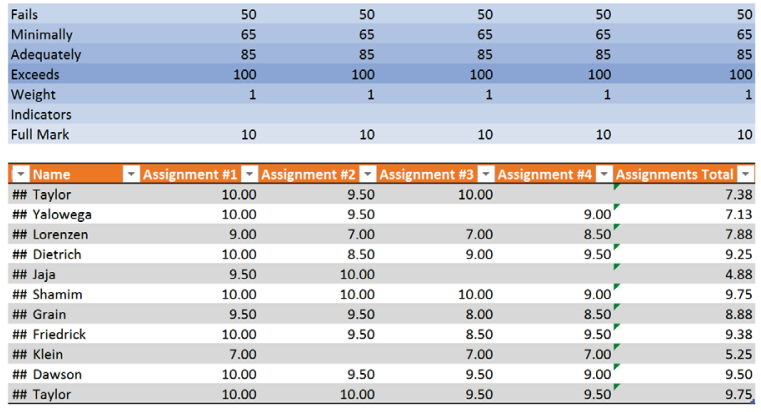

Figure 7: Grade Entry Range (Created Automatically) and the OBACIS Block

FINAL EXAM

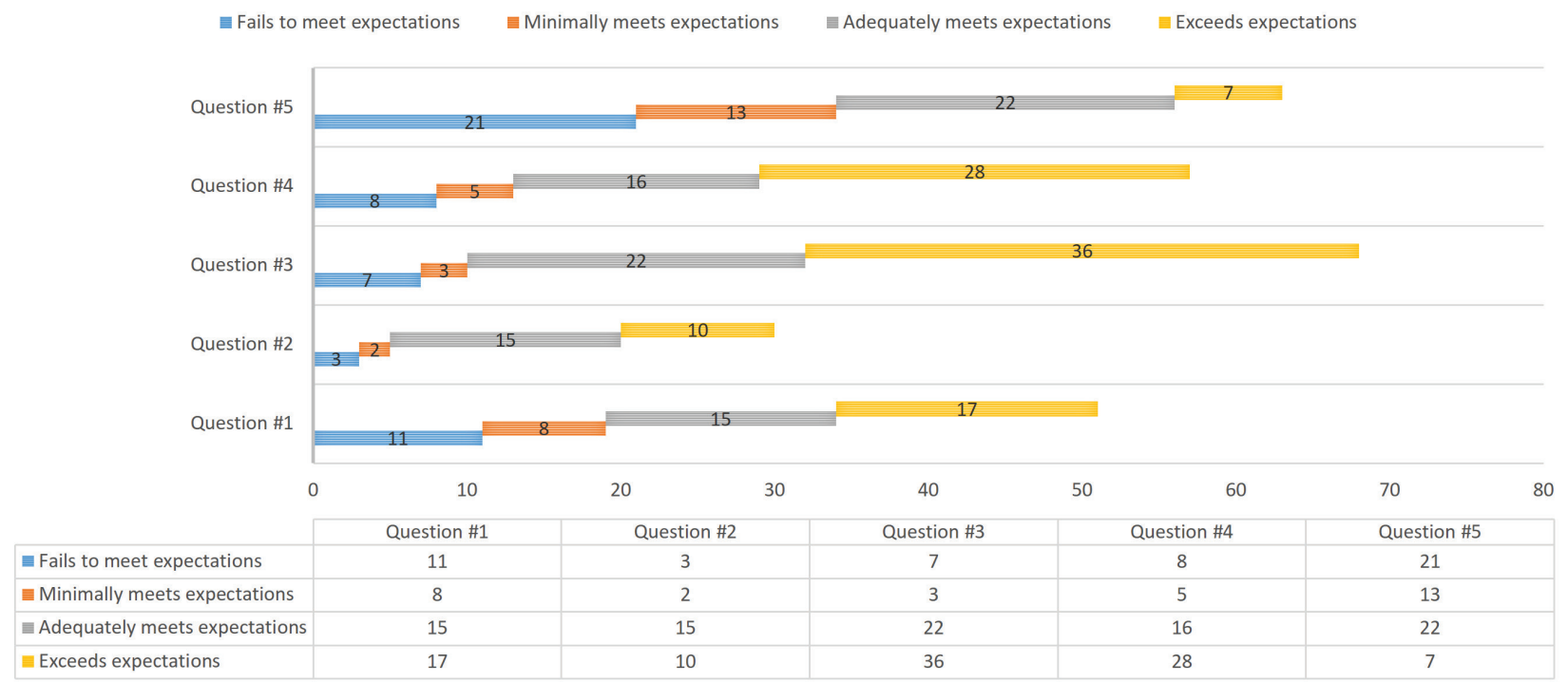

Figure 5: CA Comparative Bar Chart: Question \#5 should raise a red flag

Labs

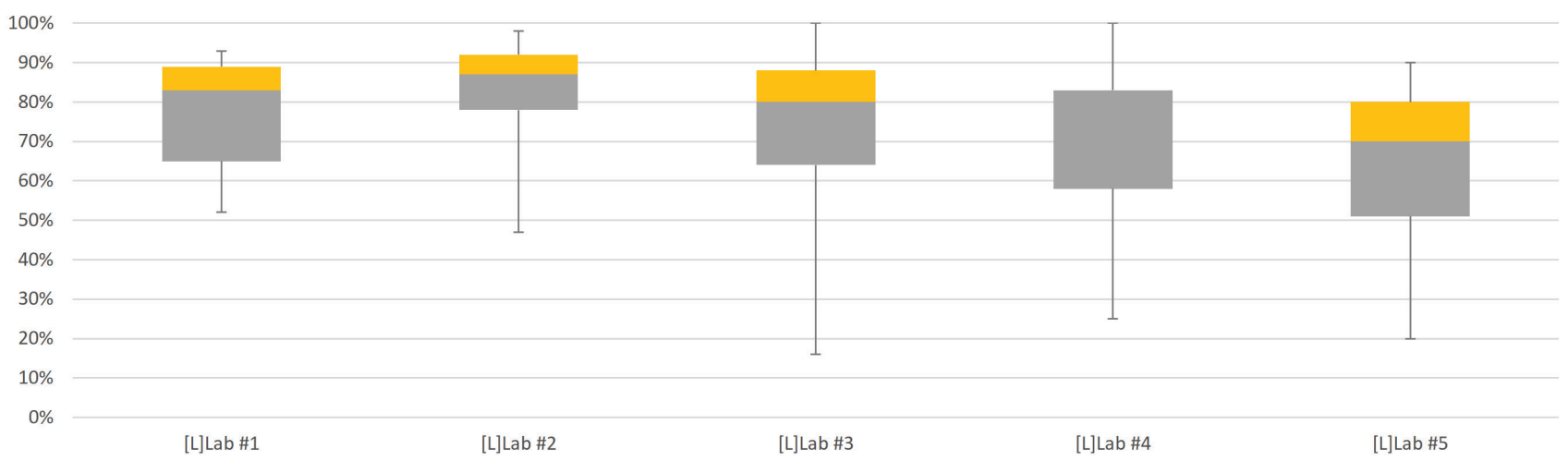

Figure 6: CA Normalized Box Plot: Lab \#5 has the lowest median, average, and highest variability

CEEA17; Paper 172

University of Toronto; June 4-7, $2017 \quad-4$ of $6-$ 


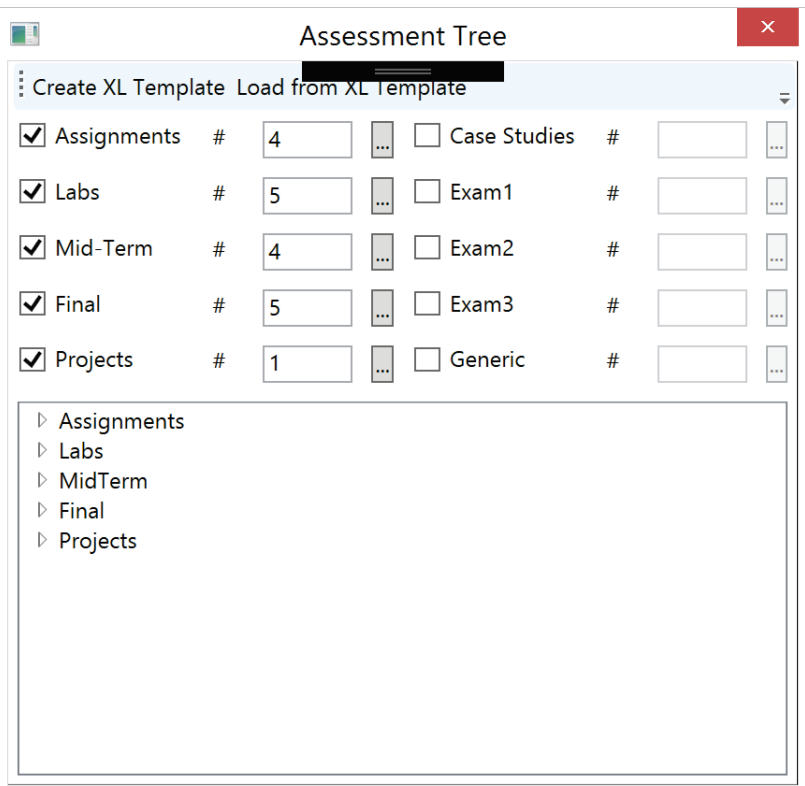

Figure 8: Assessment Tree Categories

The construction process of the assessment tree starts with defining its categories, see Figure 8 . There are 10 categories which are predefined: nine of them are predefined such as assignments, labs etc. and the last is generic. There are several grade items (assessment tools) underneath each category. If the current AGS is already defined; saved from a previous session or downloaded via the Web-App, all categories can be loaded using the "Load from Template" button. Every category has a name, weight and a set of four descriptors as mentioned earlier. By clicking the $\ldots$ button next to each

\begin{tabular}{|c|c|c|c|c|c|}
\hline 口] & \multicolumn{3}{|c|}{ Assignments Items Mapping } & $-\square$ & $x$ \\
\hline Name & & Assignments & \multirow{2}{*}{\multicolumn{2}{|c|}{$\begin{array}{l}\text { Weight } \\
\text { Minimally Acceptable \% }\end{array}$}} & 1 \\
\hline Fails \% & & 50 & & & 65 \\
\hline Adequately Accept & table \% & 85 & \multicolumn{2}{|c|}{ Exceedingly Acceptable \% } & 100 \\
\hline \multicolumn{6}{|l|}{ Grade Items } \\
\hline Name & \multicolumn{5}{|c|}{ Weight Fails Minimally Adequately Exceedingly } \\
\hline Assignment \#1 & 1 & $50 \quad 65$ & 85 & & \\
\hline Assignment \#2 & 1 & $50 \quad 65$ & 85 & & \\
\hline Assignment \#3 & 1 & $50 \quad 65$ & 85 & & \\
\hline Assignment \#4 & 1 & $50 \quad 65$ & 85 & & \\
\hline \multicolumn{6}{|c|}{ Graduate Attribute Indicators } \\
\hline \multicolumn{6}{|c|}{ Learning Outcomes } \\
\hline \multicolumn{6}{|c|}{ ID S.Name Description } \\
\hline
\end{tabular}

Figure 9: Assignments Category: Grade Descriptors, Weight, category we will tunnel down to the lower level. Figure 9 shows the dialog box that demonstrates the "Assignment category". The GA/LO panes are not updated at this stage. Assessment tools mapping are only allowed for grade items: a question in an exam, a lab report, project item, and the likes. Once the mapping is updated for grade items, those panes are

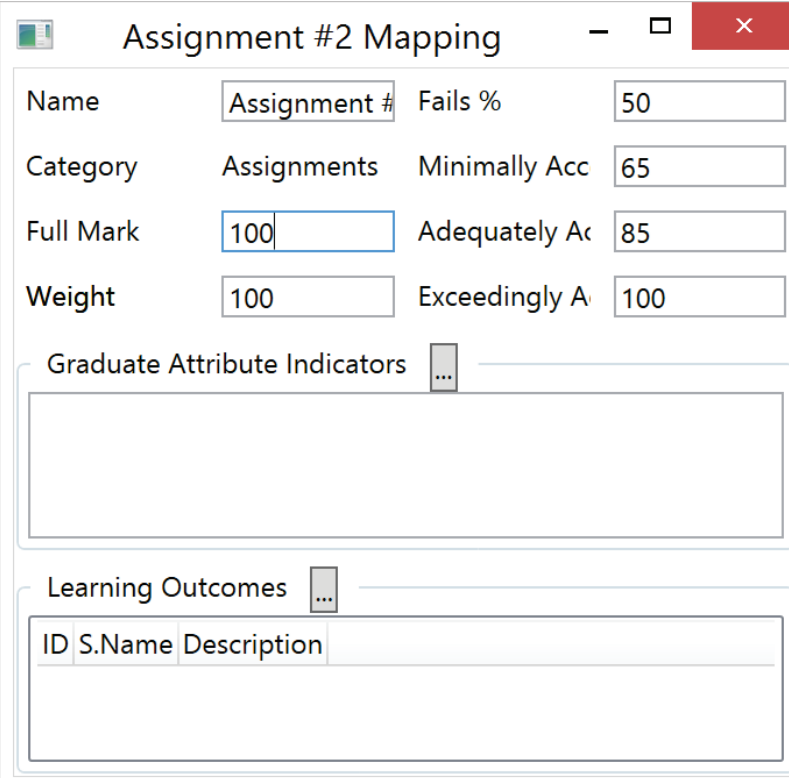

Figure 10: Assignment \#2 Grade Descriptors, Weight, ... updated automatically to list all mapped indicators or learning outcomes in those panes.

By double clicking a grade item in the list, e.g. Assignment \#2, the grade item dialog box pops out. In addition to name, weight and descriptors, the GA/LO this time have ... buttons attached to them. By clicking any of those buttons the mapping process starts. For example, if we clicked the ... button next to the GA indicators pane, the graduate attributes selector dialog appears, see Figure 11. Any number of indicators can be mapped to a grade item. Once done; by



Figure 11: Graduate Attribute Selector

closing this window, the selected indicators will be listed in GA indicators pane, see Figure 12.

All window appears in a cascading fashion as mentioned earlier, see Figure 2. A teaching faculty can go back and forth to update anything that needs change. By closing the

CEEA17; Paper 172

University of Toronto; June 4-7, 2017 
assessment tree, the underlying workbook is updated automatically.

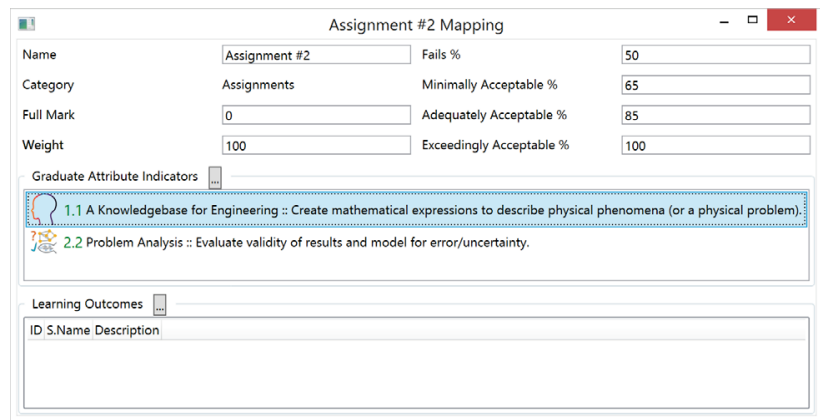

Figure 12: Assignment \#2 After Mapping GAs

Nothing needs to be done except grade entry. Once grades are updated, all reports could be updated and grades could also be exported to other systems such as LMS, Grading Systems and the likes.

\section{SUMMARY AND CONCLUSIONS}

In this paper, we introduced an Excel add-in for grade recording and accreditation reporting. The add-in is a major artifact of the OBACIS system we proposed in [9]. The Excel add-in streamlines the grade compilation and estimation processes. It provides a user-friendly interface; a set of cascading dialogs, to define the course assessment tree, OBACIS block and build the underlying workbook structure and formula needed for the OBACIS' comprehensive course reporting system. All the data generated could be uploaded to OBACIS centralized database for further processing using the Win-App to generate program and faculty level assessment reports. The app is also capable of exporting the data to other education related information systems such as learning management systems, web-marking systems and the likes.

\section{REFERENCES}

1. Kaupp, J. and B. Frank. EGAD National Snapshot Survey: Change, Progress and Improvement. in Canadian Engineering Education Association (CEEA16) 2016. Halifax, Nova Scotia

2. Dew, S.K., M. Lavoie, and A. Snelgrove, An engineering accreditation management system, in Canadian Engineering Education Association 2nd Conference 2011 June: St. John's, Newfoundland, Canada.

3. Kaupp, J., B. Frank, and C. Watts. Evaluation of software tools supporting outcomes-based continuous program improvement processes in CEEA Canadian Engineering Education Conference, CEEC13 Montreal, QC

4. Kaupp, J. and B. Frank. Evaluation of software tools supporting outcomes-based continuous program improvement processes: Part 3. in Canadian Engineering Education Conference, CEEA15. Hamilton, ON.

5. Kaupp, J. and B. Frank. Evaluation of software tools supporting outcomes-based continuous program improvement processes: Part 2. in CEEA Canadian Engineering Education Conference, CEEC14. Canmore, AB.

6. Brennan, R.W., et al. Evaluating an Integrated Course Design Tool for Engineering Graduate Attributes Assessment. in Canadian Engineering Education Association (CEEA16) 2016. Halifax, Nova Scotia

7. $\quad$ http://engineering.queensu.ca/egad. Engineering Graduate Attribute Development (EGAD) Project Last Accessed May, 2016

8. Kaupp, J. Using $R$ to Collect, Analyze and Visualize Graduate Attribute Data. in Canadian Engineering Education Association (CEEA16) 2016. Halifax, Nova Scotia

9. Ismail, M., OBACIS: Outcome Based Analytics and Continuous Improvement System, in Canadian Engineering Education Association (CEEA16)2016.

10. Ismail, M., OBACIS Phase II: Catalogs and AutoGenerated Course Information Sheets in Canadian Engineering Education Association (CEEA17)2017.

11. (CEAB), C.E.A.B., Questionnaire for Evaluation of an Engineering Program 2016.

12. (CEAB), C.E.A.B., Questionnaire for Evaluation of an Engineering Program - Exhibit 1, 2016.

CEEA17; Paper 172

University of Toronto; June 4 - 7, 2017 